\title{
Genomics of non-small cell lung cancer (NSCLC)
}

Citation for published version (APA):

Rizzo, S., Raimondi, S., de Jong, E. E. C., van Elmpt, W., De Piano, F., Petrella, F., Bagnardi, V., Jochems, A., Bellomi, M., Dingemans, A. M., \& Lambin, P. (2019). Genomics of non-small cell lung cancer (NSCLC): Association between CT-based imaging features and EGFR and K-RAS mutations in 122 patients-An external validation. European Journal of Radiology, 110, 148-155. https://doi.org/10.1016/j.ejrad.2018.11.032

Document status and date:

Published: 01/01/2019

DOI:

10.1016/j.ejrad.2018.11.032

Document Version:

Publisher's PDF, also known as Version of record

\section{Document license:}

Taverne

\section{Please check the document version of this publication:}

- A submitted manuscript is the version of the article upon submission and before peer-review. There can be important differences between the submitted version and the official published version of record.

People interested in the research are advised to contact the author for the final version of the publication, or visit the DOI to the publisher's website.

- The final author version and the galley proof are versions of the publication after peer review.

- The final published version features the final layout of the paper including the volume, issue and page numbers.

Link to publication

\footnotetext{
General rights rights.

- You may freely distribute the URL identifying the publication in the public portal. please follow below link for the End User Agreement:

www.umlib.nl/taverne-license

Take down policy

If you believe that this document breaches copyright please contact us at:

repository@maastrichtuniversity.nl

providing details and we will investigate your claim.
}

Copyright and moral rights for the publications made accessible in the public portal are retained by the authors and/or other copyright owners and it is a condition of accessing publications that users recognise and abide by the legal requirements associated with these

- Users may download and print one copy of any publication from the public portal for the purpose of private study or research.

- You may not further distribute the material or use it for any profit-making activity or commercial gain

If the publication is distributed under the terms of Article $25 \mathrm{fa}$ of the Dutch Copyright Act, indicated by the "Taverne" license above, 
Research article

\title{
Genomics of non-small cell lung cancer (NSCLC): Association between CT- based imaging features and EGFR and K-RAS mutations in 122 patients-An external validation
}

\author{
Stefania Rizzo ${ }^{\mathrm{a}, *}$, Sara Raimondi ${ }^{\mathrm{b}}$, Evelyn E.C. de Jong ${ }^{\mathrm{c}}$, Wouter van Elmpt ${ }^{\mathrm{d}}$, Francesca De Piano ${ }^{\mathrm{e}}$, \\ Francesco Petrella $^{\mathrm{f}, \mathrm{g}}$, Vincenzo Bagnardi ${ }^{\mathrm{h}}$, Arthur Jochems ${ }^{\mathrm{i}}$, Massimo Bellomi ${ }^{\mathrm{a}, \mathrm{g}}$, \\ Anne Marie Dingemans ${ }^{i}$, Philippe Lambin ${ }^{c}$ \\ ${ }^{a}$ Department of Radiology, IEO, European Institute of Oncology IRCCS, Milan, Italy \\ ${ }^{\mathrm{b}}$ Department of Epidemiology and Biostatistics, IEO, European Institute of Oncology IRCCS, Milan, Italy \\ ${ }^{\mathrm{c}}$ Department of Radiation Oncology (The D-lab), GROW-School for Oncology and Developmental Biology, Maastricht University Medical Centre, Maastricht, the \\ Netherlands \\ ${ }^{\mathrm{d}}$ Department of Radiation Oncology (MAASTRO Clinic), GROW-School for Oncology and Developmental Biology, Maastricht University Medical Centre, Maastricht, the \\ Netherlands \\ ${ }^{\mathrm{e}}$ Department of Health Sciences, University of Milan, via A. di Rudinì 8, 20142 Milan, Italy \\ ${ }^{\mathrm{f}}$ Department of Thoracic Surgery, IEO, European Institute of Oncology IRCCS, Milan, Italy \\ ${ }^{\mathrm{g}}$ Department of Oncology and Hemato-Oncology, University of Milan, Milan, Italy \\ ${ }^{\mathrm{h}}$ Department of Statistics and Quantitative Methods, University of Milano-Bicocca, Milan, Italy

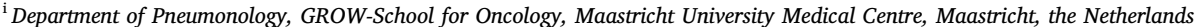

\section{A R T I C L E I N F O}

\section{Keywords:}

Lung cancer

EGF receptor

RAS proteins

Validation studies

\begin{abstract}
A B S T R A C T
Objective: To validate previously identified associations between radiological features and clinical features with Epidermal Growth Factor Receptor (EGFR)/ Kirsten RAt Sarcoma (KRAS) alterations in an independent group of patients with Non-Small Cell Lung Cancer (NSCLC).

Material and methods: A total of 122 patients with NSCLC tested for EGFR/KRAS alterations were included. Clinical and radiological features were recorded.

Univariate analysis were performed to look at the associations of the studied features with $E G F R / K R A S$ alterations. Previously calculated composite model parameters for each gene alteration prediction were applied to this validation cohort. ROC (Receiver Operating Characteristic) curves were drawn using the previously validated composite models, and also for each significant individual characteristic of the previous training cohort model. The Area Under the ROC Curve (AUC) with 95\% Confidence Intervals (CI) was calculated and compared between the full models.

Results: At univariate analysis, EGFR + confirmed an association with an internal air bronchogram, pleural retraction, emphysema and lack of smoking; KRAS + with round shape, emphysema and smoking. The AUC $(95 \% \mathrm{CI})$ in the new cohort was confirmed to be high for $E G F R+$ prediction, with a value of: $0.82(0.69-0.95)$ vs. 0.82 in the previous cohort, whereas it was smaller for KRAS + prediction, with a value of $0.60(0.48-0.72)$ vs. 0.67 in the previous cohort. Looking at single features in the new cohort, we found that the AUC for the models including only smoking was similar to that of the full model (including radiological and clinical features) for both gene alterations.

Conclusions: Although this study validated the significant association of clinical and radiological features with EGFR/KRAS alterations, models based on these composite features are not superior to smoking history alone to predict the mutations.
\end{abstract}

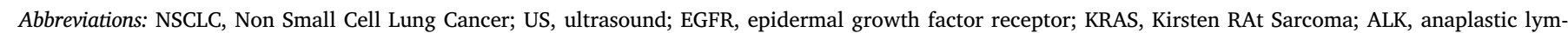

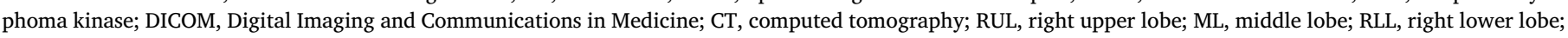

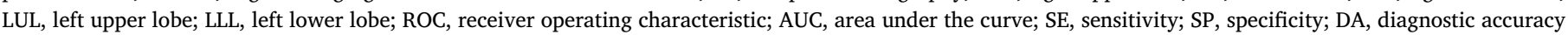

* Corresponding author.

E-mail address: stefania.rizzo@ieo.it (S. Rizzo). 


\section{Introduction}

Non-Small Cell Lung Cancer (NSCLC) accounts for more than $85 \%$ of all lung cancer cases, being the leading cause of cancer death among men and woman in the US [1]. In the last decade, the advent of novel therapeutics that specifically target signalling pathways activated by genetic alterations has revolutionized the way patients with lung cancer are treated. Recent practice guidelines in oncology and pathology recommend that all locally advanced and metastatic NSCLC with adenocarcinoma histology undergo testing for the most common targetable genetic abnormalities, such as Epidermal Growth Factor Receptor gene (EGFR) mutations and Kirsten RAt Sarcoma viral oncogene homologue (KRAS) rearrangements [2,3].

Radiomics is a high-throughput approach to translate medical images into mineable data [4,5], including quantitative features, extracted by dedicated software, and visually assessed radiological characteristics $[6,7]$. These imaging features may be then included along with clinical and genomic data in more comprehensive prognostic models $[4,5,8]$. Since the number of features is usually high, before translation into clinical practice multisite trials are needed to validate the results obtained in training cohorts on separate independent groups of patients.

A previous study focused on the radiogenomics of NSCLC in a cohort of 285 patients, and demonstrated that visually scored radiological features are associated with tumour alterations, such as EGFR, KRAS and $A L K$ mutations [6].

The purpose of this study was to validate the previously developed models for the prediction of EGFR and KRAS mutations, in an independent group of patients with lung adenocarcinoma tested for one or both of these mutations.

\section{Materials and methods}

\subsection{Patient selection}

The training set consisted of a monocentric recruitment of 285 patients with lung adenocarcinoma tested for EGFR/KRAS alterations [6]. The cohort included in this study as a validation set consisted of an external group of 122 patients from a different Institution with lung adenocarcinoma tested for EGFR mutations and KRAS rearrangements. The main inclusion criteria for both cohorts consisted of the availability of a pretreatment chest CT study including the primary tumour, and the availability of data on the EGFR mutation and/or KRAS rearrangement status. This multicentric retrospective study received approval from the Ethical Committees of both recruitment centres and an official agreement was signed by both Institutions. For the training set, written informed consent was obtained from all living patients; the local Ethical Committee approved the use of anonymized data for the other patients, waiving the informed consent (R422/16-IEO436).

\subsection{CT features evaluation}

For validation, the external centre sent the anonymised Digital Imaging and Communications in Medicine (DICOM) images of the examinations along with the clinical data specified above. Two radiologists with different degrees of experience in interpreting chest CT images, both from the centre of the training cohort, independently performed all qualitative image analyses. One radiologist was a senior radiologist with 14 years of experience in thoracic imaging (SR); the other radiologist was a fellow with three years of experience (FDP). Doubts or uncertainties of the younger radiologist (FDP) were evaluated in consensus with the senior radiologist (SR) and/or with the Head of the Division (MB).

\subsection{Data recorded}

The date of CT examinations, age, sex, EGFR and/or KRAS status were available for all patients; smoking status, when available, was recorded. For each CT scan, anonymised for personal information but unique because of the attribution of a serial number, the following data were recorded and used for statistical analysis: 1) site of the lesion, indicated as in Right Upper Lobe (RUL), Middle Lobe (ML), Right Lower Lobe (RLL), Left Upper Lobe (LUL), Left Lower Lobe (LLL), and mixed when infiltrating more than one lobe; 2) maximum diameter of the lesion in any of the three planes evaluated on the multiplanar reconstructed images with a soft tissue window; 3) shape, indicated as complex, round or oval; 4) margins, evaluated in the lung window, and indicated as smooth, lobulated or spiculated/irregular; 5) presence or absence of a peripheral ground-glass opacity; 6) density, indicated as partially solid or solid; 7) presence or absence of cavitation; 8) presence or absence of an internal air bronchogram; 9) thickening of the adjacent pleura (fissural or peripheral pleura); 10) presence or absence of intratumoral necrosis; 11) presence or absence of suspicious satellite nodules in the primary tumour lobe; 12) presence or absence of suspicious satellite nodules in non-tumour lobes; 13) presence or absence of pleural retraction; 14) location of the lesion, as central or peripheral; 15) presence or absence of nodular calcifications; 16) presence or absence of emphysema; 17) presence or absence of fibrosis; 18) presence or absence of pleural contact; 19) presence or absence of pleural effusion (Fig. 1).

\subsection{Statistical analysis}

Radiological features and clinical characteristics were expressed as mean and standard deviation for continuous variables (age and maximum diameter), and as frequency and percentage for categorical variables. The characteristics of the training and validation cohorts were compared using $t$-test for continuous variables and chi-square test for categorical variables.

The multivariate prediction models previously obtained in the training cohort [6] for EGFR and KRAS mutation/rearrangement were, respectively:

$-1.2103-0.2136 *$ (maximum diameter $[\mathrm{cm}])+0.8882 *$ (females) $+1.5168 *$ (air bronchogram) $+0.9183 *$ (pleural retraction) $-1.3695 *$ (fibrosis) -1.7150 (smoking)

and

$-2.0544+0.6071 *($ complex shape $)+0.8756 *$ (round shape) $+0.6389 *$ (nodules in non-tumour lobes) $+0.7936 *$ (smoking)

where the coefficients are multiplied by 1 if the condition is present and by 0 if the condition is absent, except for diameter, expressed in $\mathrm{cm}$.

The above parameter estimates were here applied to the validation cohort, and each patient was scored according to these models. The corresponding Receiver Operating Curves (ROC) were drawn for each gene mutation/rearrangement prediction, and the Area Under the Curve (AUC) was calculated. In the training cohort we calculated, for each gene mutation/rearrangement, the optimum cut-off that maximized Sensitivity (SE) + Specificity (SP). We then reported SE, SP and Diagnostic Accuracy (DA), with exact 95\% Confidence Intervals (CI), obtained by applying this optimum cut-off to the validation cohort.

The univariate associations of clinical and radiological features with the EGFR mutation and KRAS rearrangement in the validation cohort was evaluated by using $t$-test for continuous variables and chi-square test for categorical variables.

In order to evaluate the relative impact of each single predictor, univariate prediction models were also applied to the validation cohort and the corresponding AUC was calculated for each gene mutation/ rearrangement. Univariate and multivariable ROC curves for EGFR 


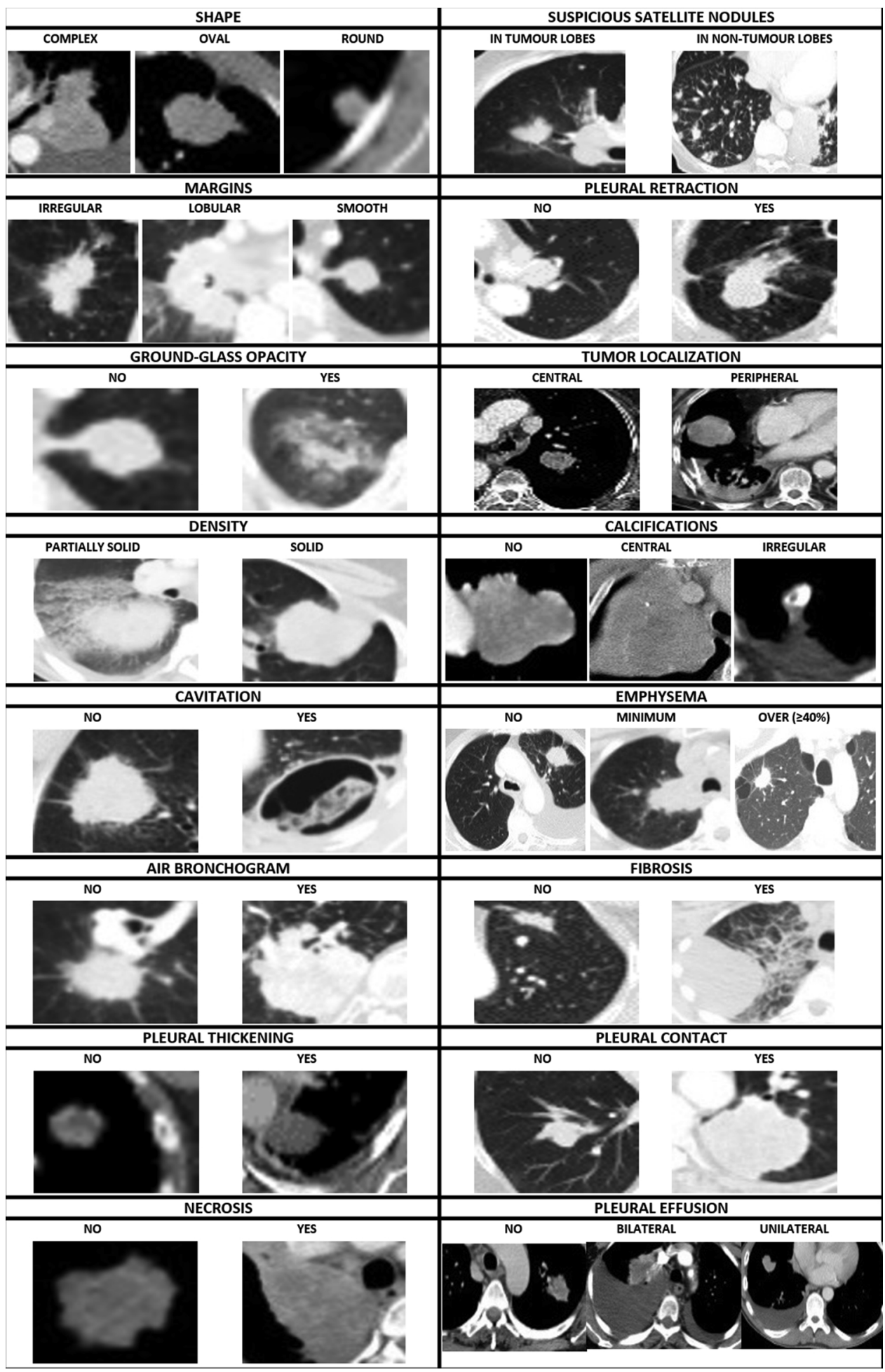

Fig. 1. Examples of the radiological qualitative features assessed.

mutations and KRAS rearrangement prediction in the validation cohort were thus compared with the test described in DeLong et al. [9] on the subset of subjects with complete data on all variables included in the full model. Calibration, which compares the predicted risk in an interval versus the average risk score in an interval, was graphically represented by calibration plots and assessed by Hosmer \& Lemeshow test. In order to have reliable risk estimates, we used five intervals (possibly including 15 patients each) to draw the calibration plot. Pvalues $<0.05$ were considered statistically significant. The analysis was performed with SAS Software (SAS Institute Inc., Cary, USA), version 9.4.

Calibration analysis was performed with $\mathrm{R}$ software, version 3.4.0.

\section{Results}

CT and clinical characteristics of the training set $(n=285)$ and validation set $(n=122)$ are included and compared in Table 1 . The training and validation sets showed significant differences for age 
Table 1

CT and clinical characteristics of the study populations in the training and validation sets.

\begin{tabular}{|c|c|c|c|}
\hline Variable & $\begin{array}{l}\text { Training set } \\
\text { N (\%) }\end{array}$ & $\begin{array}{l}\text { Validation set } \\
\mathrm{N}(\%)\end{array}$ & P-value \\
\hline Maximum diameter $(\mathrm{mm})$ & $45.49( \pm 26.27)$ & $49.78( \pm 26.45)$ & 0.14 \\
\hline Age & $65.21( \pm 9.58)$ & $61.80( \pm 11.52)$ & 0.005 \\
\hline Sex & & & 0.003 \\
\hline Male & $160 / 285(56.14 \%)$ & $49 / 122(40.16 \%)$ & \\
\hline Female & $125 / 285(44.86 \%)$ & $73 / 122(59.84 \%)$ & \\
\hline Lobe & & & 0.0002 \\
\hline RUL & $99 / 285(34.74 \%)$ & $27 / 120(22.50 \%)$ & \\
\hline ML & $16 / 285(5.61 \%)$ & $1 / 120(0.83 \%)$ & \\
\hline RLL & $40 / 285(14.04 \%)$ & $30 / 120(25.00 \%)$ & \\
\hline LUL & $83 / 285(29.12 \%)$ & $36 / 120(30.00 \%)$ & \\
\hline LLL & $42 / 285(14.74 \%)$ & $16 / 120(13.33 \%)$ & \\
\hline Mixed & $5 / 285(1.75 \%)$ & $10 / 120(8.33 \%)$ & \\
\hline Shape & & & 0.06 \\
\hline Complex & $91 / 285(31.93 \%)$ & $53 / 122(43.44 \%)$ & \\
\hline Round & $60 / 285(21.05 \%)$ & $18 / 122(14.75 \%)$ & \\
\hline Oval & $134 / 285(47.02 \%)$ & $51 / 122(41.80 \%)$ & \\
\hline Margins & & & 0.002 \\
\hline Smooth & 45/285 (15.79\%) & $17 / 122(13.93 \%)$ & \\
\hline Lobulated & 49/285 (17.19\%) & $6 / 122(4.92 \%)$ & \\
\hline Spiculated/irregular & $191 / 285(67.02 \%)$ & $99 / 122(81.15 \%)$ & \\
\hline Ground-glass opacity & $55 / 285(19.30 \%)$ & $14 / 122(11.48 \%)$ & 0.05 \\
\hline Density & & & 0.19 \\
\hline Subsolid & $33 / 283(11.66 \%)$ & $9 / 122(7.38 \%)$ & \\
\hline Solid & $250 / 283(88.34 \%)$ & $113 / 122(92.62 \%)$ & \\
\hline Cavitation & $48 / 284(16.90 \%)$ & $10 / 122(8.20 \%)$ & 0.02 \\
\hline Air bronchogram & $115 / 284(40.49 \%)$ & $43 / 122(35.25 \%)$ & 0.32 \\
\hline Thickening of the adjacent pleura & $136 / 285(47.72 \%)$ & $72 / 119(60.50 \%)$ & 0.02 \\
\hline Necrosis & $59 / 285(20.70 \%)$ & $28 / 122(22.95 \%)$ & 0.61 \\
\hline Satellite nodules in primary tumour lobe & $95 / 284(33.45 \%)$ & $48 / 122(39.34 \%)$ & 0.25 \\
\hline Nodules in non-tumour lobes & $114 / 285(40.00 \%)$ & $60 / 122(49.18 \%)$ & 0.09 \\
\hline Pleural retraction & $142 / 284(50.00 \%)$ & $94 / 122(77.05 \%)$ & $<0.0001$ \\
\hline Lesion location & & & 0.04 \\
\hline Central & $106 / 283(37.46 \%)$ & $58 / 120(48.33 \%)$ & \\
\hline Peripheral & $177 / 283(62.54 \%)$ & $62 / 120(51.67 \%)$ & \\
\hline Calcifications & $32 / 284(11.27 \%)$ & $3 / 122(2.46 \%)$ & 0.004 \\
\hline Emphysema & $52 / 285(18.25 \%)$ & $36 / 121(29.75 \%)$ & 0.01 \\
\hline Fibrosis & $36 / 284(12.68 \%)$ & $18 / 120(15.00 \%)$ & 0.53 \\
\hline Pleural contact & $181 / 285(63.51 \%)$ & $91 / 121(75.21 \%)$ & 0.02 \\
\hline Pleural effusion & $47 / 285(16.49 \%)$ & $40 / 122(32.79 \%)$ & 0.0002 \\
\hline Smoking & $133 / 261(50.96 \%)$ & $74 / 88(84.09 \%)$ & $<0.0001$ \\
\hline EGFR + & $60 / 280(21.43 \%)$ & $48 / 122(39.34 \%)$ & 0.0002 \\
\hline KRAS + & $64 / 240(26.67 \%)$ & $44 / 122(36.07 \%)$ & 0.06 \\
\hline
\end{tabular}

LLL = Left Lower Lobe; LUL = Left Upper Lobe; ML = Medium Lobe; RLL = Right Lower Lobe; RUL = Right Upper Lobe.

* T-test for continuous variables; Chi-Square test for categorical variables.

Mean ( \pm SD).

(mean \pm SD: $65.21 \pm 9.58$ and $61.80 \pm 11.52$, respectively); sex (more males in the training set and more female in the validation set; $\mathrm{M}: \mathrm{F}=160: 125$ and $\mathrm{M}: \mathrm{F}=49: 73$, respectively); location of lesion in lobes (more frequent in RUL and in LUL, respectively); central location (more frequent in the validation set); and margins (less lobulated margins in the validation set). Patients from the validation set presented more frequently with a thickening of adjacent pleura, pleural retraction, emphysema, pleural contact, pleural effusion and EGFR mutation, whereas cavitation and calcifications were less common. Smoking status information was available in the validation set only for $88 / 122(72 \%)$ patients, and it was positive for $74 / 88$ (84\%).

The ROC curve for EGFR mutation prediction in the validation set, applying the prediction model previously obtained in the training set, is shown in Fig. 2. The AUC $(95 \% \mathrm{CI})$ for the validation set was 0.82 (0.69-0.95), as the AUC of the training set (0.82 [6]). The correspondent calibration plot is shown in Supplementary Fig. 1. With a cut-off for the model score of $-1.21923,14 / 20 E G F R+$ patients in the validation cohort were correctly identified (SE; 95\%CI $=70 \%$; 46-88\%) and 52/63 EGFR- patients in the validation cohort were correctly identified $(\mathrm{SP} ; 95 \% \mathrm{CI}=83 \% ; 71-91 \%)$, with $\mathrm{DA} \quad(95 \% \mathrm{CI})=80 \%$ (69-88\%).

The ROC curve for KRAS rearrangement prediction in the validation set, applying the prediction model previously obtained in the training set, is shown in Fig. 3. The AUC $(95 \% \mathrm{CI})$ for the validation set is 0.60 $(0.48-0.72)$, slightly smaller than that calculated in the training set (0.67 [6]).

The correspondent calibration plot is shown in Supplementary Fig. 2. With a cut-off for the model score of $-0.8384,32 / 41$ KRAS + patients in the validation cohort were correctly identified (SE; $95 \% \mathrm{CI}=78 \% ; 62-89 \%)$ and $17 / 47 \mathrm{KRAS}-$ patients in the validation cohort were correctly identified (SP; 95\%CI $=36 \%$; 23-51\%), with DA $(95 \% \mathrm{CI})=56 \%(45-66 \%)$.

The univariate association of CT and clinical features with the EGFR mutation in the validation set is shown in Table 2. Compared to the training set [6], the EGFR + mutation confirmed an association with an internal air bronchogram, pleural retraction, and absence of emphysema and of smoking. Smoking was the most associated factor, with almost all EGFR- patients being smokers $(65 / 67 ; 97 \%)$ compared to 9 smokers out of $21 E G F R+$ patients (43\%).

Fig. 4 shows ROC curves for EGFR mutation prediction in the validation set, showing that, as previously demonstrated in the training set, the AUC for the full model (including both radiological and clinical features) is significantly higher than that of each characteristic ( $p<0.003$ for all comparisons) except smoking ( $p=0.59$ ). 


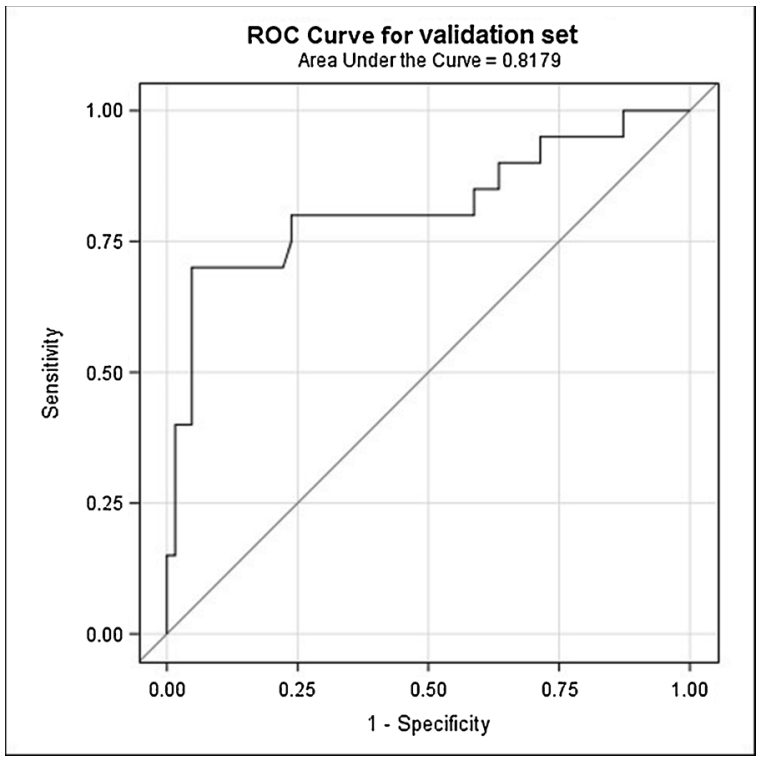

Fig. 2. ROC curve for EGFR mutation prediction in the validation set, applying the prediction model of the training set [6].

Note: Area Under the Curve (AUC) for the training cohort was 0.82 [6]; AUC $(95 \% \mathrm{CI})$ for the validation cohort $=0.82(0.69-0.95)$.

* $-1.2103-0.2136 *$ (maximum diameter $[\mathrm{cm}])+0.8882 *$ (females) $+1.5168 *$ (air bronchogram) $+0.9183^{*}$ (pleural retraction)-1.3695*(fibrosis)-1.7150(smoking). Except for diameter, coefficients were multiplied by 1 if the condition was present and by 0 if the condition was absent.

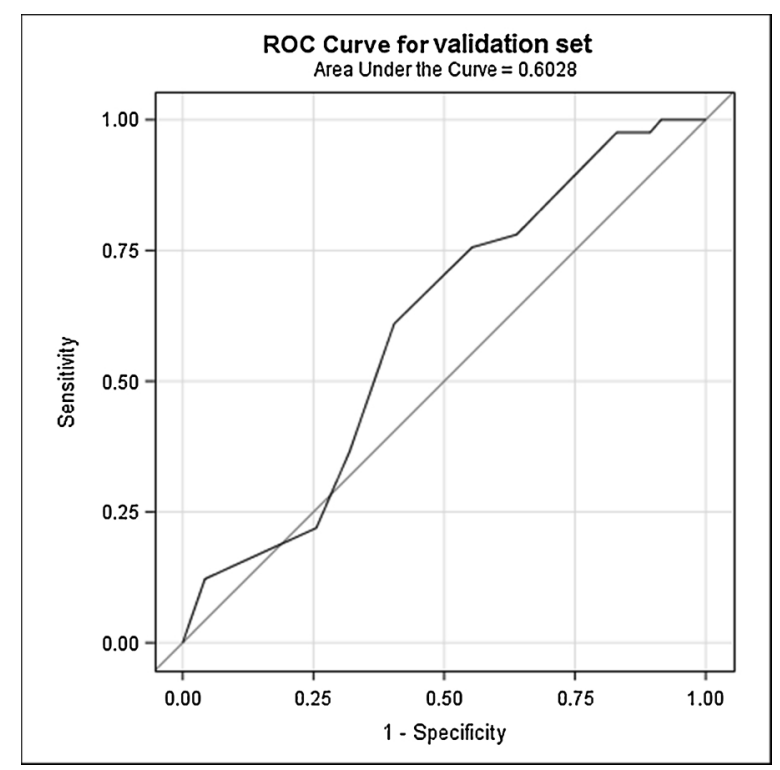

Fig. 3. ROC curve for KRAS mutation prediction in the validation set, applying the prediction model of the training set [6].

Note: Area Under the Curve for the training cohort was 0.67 [6]; AUC (95\%CI) for the validation cohort $=0.60(0.48-0.72)$

$*-2.0544+0.6071 *$ (complex shape) $+0.8756 *$ (round shape) $+0.6389 *$ (nodules in non-tumour lobes) $+0.7936^{*}$ (smoking). Coefficients were multiplied by 1 if the condition was present and by 0 if the condition was absent.

Univariate association for CT and clinical features with the KRAS rearrangement in the validation set are listed in Table 3. At univariate analysis KRAS + rearrangement confirmed an association with round shape, emphysema and smoking. Smoking was the most associated factor, with almost all KRAS + patients being smokers (39/41; 95\%) compared to 35 smokers out of 47 KRAS- patients (74\%).

Fig. 5 shows ROC curves for KRAS rearrangement prediction in the
Table 2

Association of patient, tumour and CT features with the EGFR mutation in the validation set: univariate analysis.

\begin{tabular}{|c|c|c|c|}
\hline & \multicolumn{3}{|l|}{ EGFR } \\
\hline & $-\mathrm{N}(\%)$ & + N (\%) & $P$ value \\
\hline Maximum diameter & $\begin{array}{l}48.82 \\
( \pm 28.76)\end{array}$ & $\begin{array}{l}51.26 \\
( \pm 22.66)\end{array}$ & 0.63 \\
\hline Age & $60.74( \pm 1.04)$ & $\begin{array}{l}63.44 \\
( \pm 12.17)\end{array}$ & 0.21 \\
\hline Sex & & & 0.11 \\
\hline Male & $34(46 \%)$ & $15(31 \%)$ & \\
\hline Female & $40(54 \%)$ & $33(69 \%)$ & \\
\hline Lobe & & & 0.40 \\
\hline LSD & $15(21 \%)$ & $12(25 \%)$ & \\
\hline LM & $0(0 \%)$ & $1(2 \%)$ & \\
\hline LID & $15(21 \%)$ & $15(31 \%)$ & \\
\hline LSS & $23(32 \%)$ & $13(27 \%)$ & \\
\hline LIS & $12(17 \%)$ & $4(8 \%)$ & \\
\hline Mixed & $7(10 \%)$ & $3(6 \%)$ & \\
\hline Shape & & & 0.006 \\
\hline Complex & $29(39 \%)$ & $24(50 \%)$ & \\
\hline Round & $17(23 \%)$ & $1(2 \%)$ & \\
\hline Oval & $28(38 \%)$ & $23(48 \%)$ & \\
\hline Margins & & & 0.45 \\
\hline Smooth & $11(15 \%)$ & $6(13 \%)$ & \\
\hline Lobulated & $5(7 \%)$ & $1(2 \%)$ & \\
\hline Spiculated/irregular & $58(78 \%)$ & $41(85 \%)$ & \\
\hline Peripheral halo & $11(15 \%)$ & $3(6 \%)$ & 0.14 \\
\hline Density & & & 0.07 \\
\hline Partially solid & $8(11 \%)$ & $1(2 \%)$ & \\
\hline Solid & $66(89 \%)$ & $47(98 \%)$ & \\
\hline Cavitation & $6(8 \%)$ & $4(8 \%)$ & 0.96 \\
\hline Internal air bronchogram & $21(28 \%)$ & $22(46 \%)$ & 0.05 \\
\hline Thickening of the adjacent pleura & $38(52 \%)$ & $34(74 \%)$ & 0.02 \\
\hline Necrosis & $15(20 \%)$ & $13(27 \%)$ & 0.38 \\
\hline Satellite nodules in primary tumour lobe & $27(36 \%)$ & $21(44 \%)$ & 0.42 \\
\hline Nodules in non-tumour lobes & $35(47 \%)$ & $25(52 \%)$ & 0.61 \\
\hline Pleural retraction & $52(70 \%)$ & $42(88 \%)$ & 0.03 \\
\hline Lesion location & & & 0.30 \\
\hline Central & $32(44 \%)$ & $26(54 \%)$ & \\
\hline Peripheral & $40(56 \%)$ & $22(46 \%)$ & \\
\hline Calcifications & $2(3 \%)$ & $1(2 \%)$ & 0.83 \\
\hline Emphysema & $31(42 \%)$ & $5(10 \%)$ & 0.0002 \\
\hline Fibrosis & $13(18 \%)$ & $5(10 \%)$ & 0.25 \\
\hline Pleural contact & $52(71 \%)$ & $39(81 \%)$ & 0.21 \\
\hline Pleural effusion & $22(30 \%)$ & $18(38 \%)$ & 0.37 \\
\hline Smoking $^{\infty}$ & $65(97 \%)$ & $9(43 \%)$ & $<0.0001$ \\
\hline
\end{tabular}

$\mathrm{CI}=$ Confidence Interval; LLL = Left Lower Lobe; LUL = Left Upper Lobe; $\mathrm{ML}=$ Medium Lobe; RLL $=$ Right Lower Lobe; RUL $=$ Right Upper Lobe.

Note: significant ORs and p-values are in bold.

* T-test for continuous variables; Chi-Square test for categorical variables.

Mean ( \pm SD)

${ }^{\infty}$ Smoking data available for $\mathrm{N}=67$ EGFR- and $\mathrm{N}=21$ EGFR + patients.

validation set, showing that, as previously demonstrated in the training set, the AUC for the full model was higher than that of nodules in nontumour lobes $(p=0.05)$, whereas it was similar to that for shape $(\mathrm{p}=0.24)$ and smoking $(\mathrm{p}=0.99)$.

\section{Discussion}

This study validated the performance of a model based on radiological features and clinical features for prediction of EGFR mutation and/or KRAS rearrangement in an independent external group of 122 lung cancer patients (validation set) [6].

The radiomic process can rely on the evaluation of quantitative features, radiological qualitative features, or both [4]. On the one hand, the development of different software to extract quantitative features from digital images in DICOM format [10] has aroused growing interest in the field of radiomics, with a focus on quantitative features. On the other, previous studies have demonstrated that radiological features, 


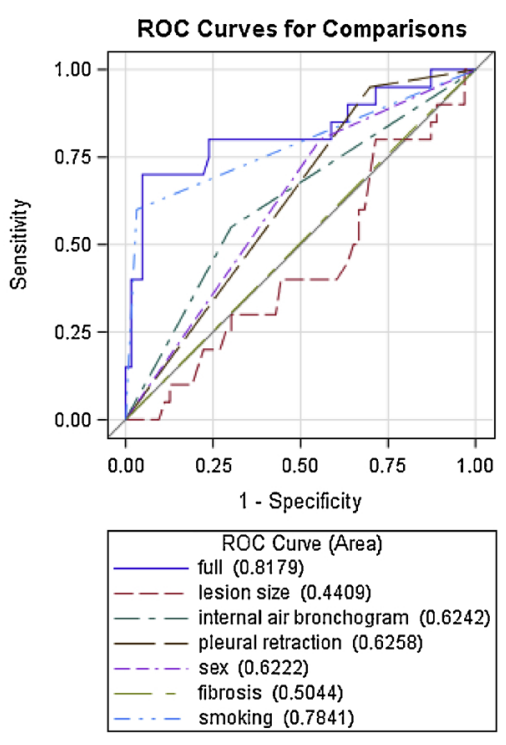

Fig. 4. ROC curves for EGFR mutation prediction between each characteristic significantly associated with EGFR in the validation set.

*estimates obtained in the training cohort are applied

corresponding to descriptive features more familiar for radiologists, may be associated with tumour biology [6]. Subsequent studies further demonstrated that the combination of radiological qualitative and quantitative features, and the inclusion of clinical information strengthens the robustness of radiomic models [4].

Validation of a model in an external cohort of patients gives robustness and consistency to the model itself in all cases [11]. Based on previous papers, a reasonable number of patients to be included in a radiomics analysis should be ten for each feature in a model based on binary classifiers [4], thus large cohorts of patients for training and validation studies are needed. Moreover, it is well known that model fitting is optimal in the training set used to build the model itself, while validation in an external cohort provides more reliable fitting estimates [12]. Finally, external validation will determine the transportability of the model in different locations consisting of plausibly similar individuals (different case-mix) [13]. Accordingly, the sharing of image data among different centres is appropriate and valuable. The best models are those that can accommodate additional clinical or genomic covariates, along with radiological and quantitative features, taking into account differences in acquisitions, protocols and patients' populations $[4,8,14-16]$.

This study validated a model for prediction of the EGFR mutation and KRAS rearrangement in NSCLC, based on radiological and clinical features. In the training cohort, we found that an air bronchogram, pleural retraction, and small size (radiological features), were associated with the EGFR mutation [6]. In the same cohort major clinical features, concordant with data already known in the literature [17], were: sex (females more frequently mutated than males), smoking history (non smokers more frequently mutated than smokers), emphysema and fibrosis (both less frequent in mutated patients than in nonmutated ones).

As shown in the results section, for EGFR mutation prediction, the AUC for our validation set was $0.82(0.69-0.95)$. This result shows a better performance of the model presented by a previous study where discrimination between EGFR + and EGFR-, based on a radiomic quantitative signature, showed an AUC $=0.69$, although it is of note that the authors demonstrated an improvement of the performance when they combined the radiomic signature with a clinical model (AUC $=0.75)$ [18]. Likewise, Liu et al demonstrated that a multiple logistic regression model adding radiomic features to a clinical model
Table 3

Association of CT and clinical features with the KRAS mutation in the validation set: univariate analysis.

\begin{tabular}{|c|c|c|c|}
\hline & \multicolumn{3}{|l|}{ KRAS } \\
\hline & $\begin{array}{l}- \\
\mathrm{N}(\%)\end{array}$ & $\begin{array}{l}+ \\
\mathrm{N}(\%)\end{array}$ & Pvalue \\
\hline Maximum diameter & $\begin{array}{l}48.82 \\
( \pm 28.76)\end{array}$ & $\begin{array}{l}51.26 \\
( \pm 22.66)\end{array}$ & 0.49 \\
\hline Age & $\begin{array}{l}60.74 \\
( \pm 11.04)\end{array}$ & $\begin{array}{l}63.44 \\
( \pm 12.17)\end{array}$ & 0.15 \\
\hline Sex & & & 0.30 \\
\hline Male & $34(44 \%)$ & $15(34 \%)$ & \\
\hline Female & $44(56 \%)$ & $29(66 \%)$ & \\
\hline Lobe & & & 0.46 \\
\hline LSD & $21(27 \%)$ & $6(14 \%)$ & \\
\hline LM & $1(1 \%)$ & $0(0 \%)$ & \\
\hline LID & $20(26 \%)$ & $10(23 \%)$ & \\
\hline LSS & $21(27 \%)$ & $15(35 \%)$ & \\
\hline LIS & $9(12 \%)$ & $7(16 \%)$ & \\
\hline Mixed & $5(6 \%)$ & $5(12 \%)$ & \\
\hline Shape & & & 0.009 \\
\hline Complex & $39(50 \%)$ & $14(32 \%)$ & \\
\hline Round & $6(8 \%)$ & $12(27 \%)$ & \\
\hline Oval & $33(42 \%)$ & $18(41 \%)$ & \\
\hline Margins & & & 0.23 \\
\hline Smooth & $10(13 \%)$ & $7(16 \%)$ & \\
\hline Lobulated & $2(3 \%)$ & $4(9 \%)$ & \\
\hline Spiculated/irregular & $66(85 \%)$ & $33(75 \%)$ & \\
\hline Peripheral halo & $7(9 \%)$ & $7(16 \%)$ & 0.25 \\
\hline Density & & & 0.21 \\
\hline Partially solid & $4(5 \%)$ & $5(11 \%)$ & \\
\hline Solid & $74(95 \%)$ & $39(89 \%)$ & \\
\hline Cavitation & $7(9 \%)$ & $3(7 \%)$ & 0.68 \\
\hline Internal air bronchogram & $30(38 \%)$ & $13(30 \%)$ & 0.32 \\
\hline Thickening of the adjacent pleura & $50(67 \%)$ & $22(50 \%)$ & 0.07 \\
\hline Necrosis & $16(21 \%)$ & $12(27 \%)$ & 0.39 \\
\hline Satellite nodules in primary tumour lobe & $34(44 \%)$ & $14(32 \%)$ & 0.20 \\
\hline Nodules in non-tumour lobes & $38(49 \%)$ & $22(50 \%)$ & 0.89 \\
\hline Pleural retraction & $63(81 \%)$ & $31(70 \%)$ & 0.19 \\
\hline Lesion location & & & 0.10 \\
\hline Central & $42(54 \%)$ & $16(38 \%)$ & \\
\hline Peripheral & $36(46 \%)$ & $26(62 \%)$ & \\
\hline Calcifications & $2(3 \%)$ & $1(2 \%)$ & 0.92 \\
\hline Emphysema & $18(23 \%)$ & $18(42 \%)$ & 0.03 \\
\hline Fibrosis & $11(14 \%)$ & $7(16 \%)$ & 0.77 \\
\hline Pleural contact & $59(76 \%)$ & $32(74 \%)$ & 0.88 \\
\hline Pleural effusion & $30(38 \%)$ & $10(23 \%)$ & 0.08 \\
\hline Smoking ${ }^{\infty}$ & $35(74 \%)$ & $39(95 \%)$ & 0.008 \\
\hline
\end{tabular}

$\mathrm{CI}=$ Confidence Interval; LLL $=$ Left Lower Lobe; LUL = Left Upper Lobe; $\mathrm{ML}=$ Medium Lobe; RLL = Right Lower Lobe; RUL = Right Upper Lobe.

Note: significant ORs and p-values are in bold.

* T-test for continuous variables; Chi-Square test for categorical variables. Mean ( \pm SD).

${ }^{\infty}$ Smoking data available for $\mathrm{N}=47 \mathrm{KRAS}$ - and $\mathrm{N}=41 \mathrm{KRAS}+$ patients.

resulted in a significant improvement of predicting power, by increasing the AUC from 0.667 to $0.709(\mathrm{P}<.0001)$ [19]. Despite the differences in the overall performance of the models, that may require further validations, it is clear that the combination of clinical and radiological features improves the output of all the model assessed.

In our validation cohort, internal air bronchogram and pleural retraction (radiological features), along with absence of emphysema and of smoking (clinical features), were significantly associated with the $E G F R+$ mutation. Indeed, when using in the validation cohort the same cutoff for the model score of -1.21923 used in the training cohort, the SE, SP and DA were $70 \%, 83 \%$ and $80 \%$ respectively, thus confirming, although a DA of $80 \%$ is still not perfect, the importance of the abovementioned radiological features for association with EGFR+ mutation. In the validation cohort, a major role was observed for smoking history: the prediction accuracy for the model including only smoking data was similar to that of the full model including both 


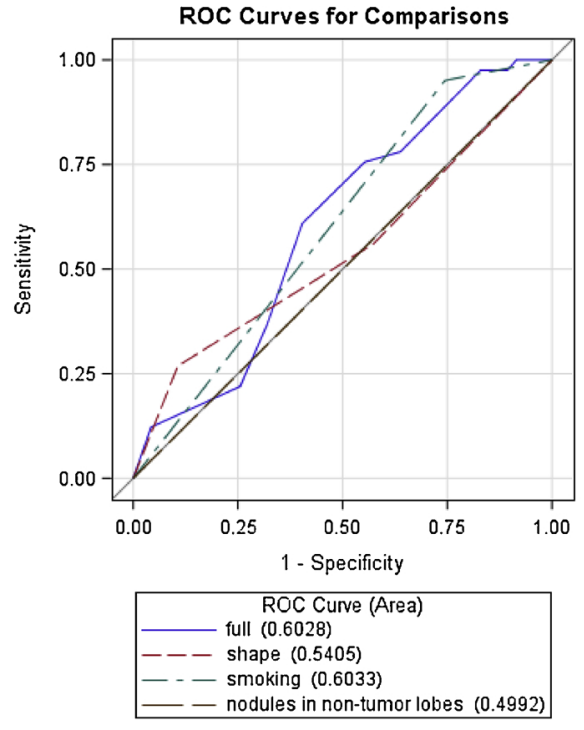

Fig. 5. ROC curves for KRAS mutation prediction between each characteristic significantly associated with KRAS in the validation set.

*estimates obtained in the training cohort are applied

clinical and CT features. This is likely due to the fact that in the validation set smoking history was positive for the large majority of the subjects with available data (84\%), with a possibility of oversampling of smokers in this cohort. Because of the known association between smoking and EGFR [20] this oversampling may "conceal" the effect of other possible predictors.

In the training cohort, as well as in the validation cohort, the presence of an air bronchogram was significantly associated with the presence of EGFR mutation. This result is concordant with previous studies demonstrating that effective EGFR mutations are frequently accompanied by air bronchogram [6,21,22].

In this study, we validated the association between pleural retraction and EGFR mutation. This result is in agreement with previous studies, where multivariable regression analyses indicated that pleural retraction was one of the prognostic factors for harboring EGFR mutation [23].

In the training cohort there was a significant association between small size and EGFR, that was concordant with other studies [24]. However, in the validation cohort, the association between small lesion diameter and EGFR + mutation was not confirmed at the univariate analysis. This might be due to the different type of accrual of the two cohorts. Indeed, the training cohort was selected in an oncological hospital, according to the availability of assessment of gene mutations or rearrangements, regardless of cancer stage. Instead, the validation cohort included patients from hospitals in the southern region of the Netherlands, mainly with advanced lung cancer (because in the Netherlands only patients with stage IIIb or IV receiving a palliative treatment are tested for EGFR or KRAS mutations).

The absence of emphysema was confirmed as feature associated with presence of EGFR mutation in the training cohort as well as in the validation cohort. These results are concordant with other studies in the literature where the EGFR mutation group was not prone to emphysema $(p=0.002)[24,25]$, as well as in agreement with other authors that quantified 30 detailed CT features, demonstrating that severe peripheral emphysema was more likely associated with wild-type EGFR tumors [22].

The model for prediction of the KRAS rearrangement in the training cohort demonstrated a significant association with radiological features, such as round shape and nodules in non-tumour lobes, along with smoking history. In the validation cohort, univariate analysis confirmed the association of the KRAS + rearrangement with round lesion shape and smoking history, whereas the association with nodules in non-tumour lobes was not confirmed. As mentioned in the analysis of the training cohort, the statistical significance of nodules in non-tumour lobes was close to unity, therefore this association needed a validation [6]. Nodules in non-tumour lobes refer to a haematogenous dissemination of cells, usually seen in more aggressive tumours. Since the KRAS rearrangement is associated with a worse prognosis $[25,26]$, this may coexist with haematogenous dissemination, and not necessarily with the KRAS rearrangement. In the validation group, smoking history confirmed its association with the KRAS + rearrangement, as known in the literature $[27,28]$.

As for $E G F R+$ prediction, the full model comparing the ROC curves for association of features and KRAS + rearrangement did not show a significant improvement when compared to a model based only on round shape or smoking.

This study has some limitations. The main drawback is the lack of detailed information on smoking history in the validation group. Indeed, smoking was an important feature in the model built on the training cohort, but its importance was overestimated in the validation group, where the majority of patients were smokers. However, it does not affect the univariate analysis, where each single feature has its own importance, and the main associations of radiological and clinical features with the EGFR + mutation and KRAS + rearrangement were confirmed. Another limitation is the lack of uniformity in the selection of patients, and the relative lack of stratification for stages. As already mentioned, this might have influenced the lack of significance of small size for lesion association with EGFR. However, the aim of this study was to validate a prognostic model for prediction of the $E G F R+\mathrm{mu}$ tation and KRAS + rearrangement, irrespective of disease stage and therapy.

In conclusion, this study validated in NSCLC patients the association of radiological features (internal air bronchogram,and pleural retraction) and clinical features (lack of emphysema and smoking) with the $E G F R+$ mutation and of round shape and smoking with KRAS mutation. The weight of smoking history alone is however as important as that of imaging features.

\section{Conflict of interest}

None.

\section{Acknowledgements}

The English text was edited by Anne Prudence Collins (Editor and Translator Medical \& Scientific Publications). Patients' data were managed by Cristiana Fodor (Data Manager; Scientific Direction, European Institute of Oncology, Milan IT).

PL and EdJ acknowledge financial support from ERC advanced grant (ERC-ADG-2015, $\mathrm{n}^{\circ} 694812$ - Hypoximmuno) and the Dutch technology Foundation STW (grant n ${ }^{\circ}$ P14-19 Radiomics STRaTegy), which is the applied science division of NWO, and the Technology Programme of the Ministry of Economic Affairs.

\section{Appendix A. Supplementary data}

Supplementary material related to this article can be found, in the online version, at doi:https://doi.org/10.1016/j.ejrad.2018.11.032.

\section{References}

[1] R.L. Siegel, K.D. Miller, A. Jemal, Cancer statistics, 2018, CA Cancer J. Clin. 68 (1) (2018) 7-30.

[2] AIOT guidelines at http://www.oncologiatoracica.it/linee3/231.pdf.

[3] National Comprehensive Cancer Network NCCN guidelines: Non-Small Cell Lung Cancer (Version 7.2015), (2018) http://www.nccn.org/professionals/physician. gls/pdf/nscl.pdf. 
[4] R.J. Gillies, P.E. Kinahan, H. Hricak, Radiomics: images are more than pictures, they are data, Radiology 278 (2) (2016) 563-577.

[5] P. Lambin, E. Rios-Velazquez, R. Leijenaar, et al., Radiomics: extracting more information from medical images using advanced feature analysis, Eur. J. Cancer 48 (4) (2012) 441-446.

[6] S. Rizzo, F. Petrella, V. Buscarino, et al., CT radiogenomic characterization of EGFR, K-RAS, and ALK mutations in non-small cell lung cancer, Eur. Radiol. 26 (1) (2016) $32-42$.

[7] S. Hawkins, H. Wang, Y. Liu, et al., Predicting malignant nodules from screening CT scans, J. Thorac. Oncol. 11 (12) (2016) 2120-2128.

[8] E.J. Limkin, R. Sun, L. Dercle, et al., Promises and challenges for the implementation of computational medical imaging (radiomics) in oncology, Ann. Oncol. 28 (6) (2017) 1191-1206.

[9] E.R. DeLong, D.M. DeLong, D.L. Clarke-Pearson, Comparing the areas under two or more correlated receiver operating characteristic curves: a nonparametric approach, Biometrics 44 (3) (1988) 837-845.

[10] R.T. Larue, G. Defraene, D. De Ruysscher, P. Lambin, W. van Elmpt, Quantitative radiomics studies for tissue characterization: a review of technology and methodological procedures, Br. J. Radiol. 90 (1070) (2017) 20160665.

[11] G.S. Collins, J.B. Reitsma, D.G. Altman, K.G. Moons, Transparent reporting of a multivariable prediction model for individual prognosis or diagnosis (TRIPOD), Br. J. Surg. 102 (3) (2015) 148-158.

[12] F.E. Harrell Jr, K.L. Lee, D.B. Mark, Multivariable prognostic models: issues in developing models, evaluating assumptions and adequacy, and measuring and reducing errors, Stat. Med. 15 (4) (1996) 361-387 28.

[13] G.S. Collins, J.A. de Groot, S. Dutton, et al., External validation of multivariable prediction models: a systematic review of methodological conduct and reporting, BMC Med. Res. Methodol. 14 (2014) 40.

[14] T. Dalal, M.K. Kalra, S. Rizzo, et al., Metallic prosthesis: technique to avoid increase in CT radiation dose with automatic tube current modulation in a phantom and patients, Radiology 236 (2) (2005) 671-675.

[15] P. Lambin, R.T.H. Leijenaar, T.M. Deist, et al., Radiomics: the bridge between medical imaging and personalized medicine, Nat. Rev. Clin. Oncol. 14 (12) (2017) $749-762$.

[16] S. Rizzo, F. Botta, S. Raimondi, et al., Radiomics of high-grade serous ovarian cancer: association between quantitative CT features, residual tumour and disease progression within 12 months, Eur. Radiol. (May 8) (2018), https://doi.org/10. 1007/s00330-018-5389-z.

[17] T.J. Lynch, D.W. Bell, R. Sordella, et al., Activating mutations in the EGFR receptor underlying responsiveness of non-small-cell lung cancer to gefitinib, N. Engl. J. Med. 350 (2004) 2129-2139.

[18] E. Rios Velazquez, C. Parmar, Y. Liu, T.P. Coroller, et al., Somatic mutations drive distinct imaging phenotypes in lung cancer, Cancer Res. 77 (14) (2017) 3922-3930.

[19] Y. Liu, J. Kim, Y. Balagurunathan, et al., Radiomic features are associated with EGFR mutation status in lung adenocarcinomas, Clin. Lung Cancer 17 (5) (2016) 441-448 e6.

[20] J.H. Ren, W.S. He, G.L. Yan, M. Jin, K.Y. Yang, Wu G EGFR mutations in non-smallcell lung cancer among smokers and non-smokers: a meta-analysis, Environ. Mol. Mutagen. 53 (1) (2012) 78-82.

[21] J. Dai, J. Shi, A.K. Soodeen-Lalloo, et al., Air bronchogram: a potential indicator of epidermal growth factor receptor mutation in pulmonary subsolid nodules, Lung Cancer 98 (2016) 22-28.

[22] J. Lv, H. Zhang, J. Ma, et al., Comparison of CT radiogenomic and clinical characteristics between EGFR and KRAS mutations in lung adenocarcinomas, Clin. Radiol. 73 (6) (2018) 590.e1-590.e8.

[23] Y. Liu, J. Kim, F. Qu, et al., CT features associated with epidermal growth factor receptor mutation status in patients with lung adenocarcinoma, Radiology 280 (2016) 271-280.

[24] J.S. Hsu, M.S. Huang, C.Y. Chen, et al., Correlation between EGFR mutation status and computed tomography features in patients with advanced pulmonary adenocarcinoma, J. Thorac. Imaging 29 (2014) 357-363.

[25] M. Del Re, E. Rofi, G. Restante, et al., Implications of KRAS mutations in acquired resistance to treatment in NSCLC, Oncotarget 9 (5) (2017) 6630-6643.

[26] M. Román, I. Baraibar, I. López, et al., KRAS oncogene in non-small cell lung cancer: clinical perspectives on the treatment of an old target, Mol. Cancer 17 (1) (2018) 33.

[27] G.J. Riely, M.G. Kris, D. Rosenbaum, et al., Frequency and distinctive spectrum of KRAS mutations in never smokers with lung adenocarcinoma, Clin. Cancer Res. 14 (2008) 5731-5734.

[28] S.H. Ou, Lung cancer in never-smokers. Does smoking history matter in the era of molecular diagnostics and targeted therapy? J. Clin. Pathol. 66 (10) (2013) 839-846. 